\title{
The incidence and predictors of pneumothorax among trauma patients in Saudi Arabia
}

\author{
Findings from a level-I trauma center
}

Suliman Alghnam, PhD, MHA, Mahdya H. Aldahnim, RT, Mohammed H. Aldebasi, Medical Student,

Jawaher A. Towhari, Medical Student, Abdulkareem S. Alghamdi, Medical Student, Alanood A. Alharbi, Medical Student, Yabya A. Almarhabi, MD, Ibrabim T. Albabtain, MD.

\begin{abstract}

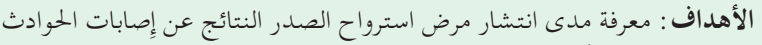

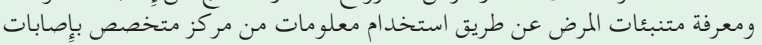
حوادث الدرجة الأولى. - معن.

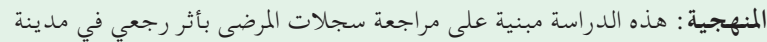

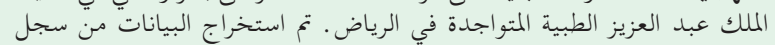

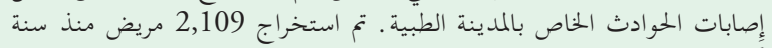

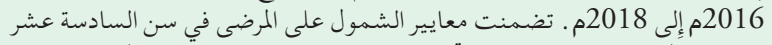

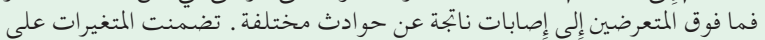

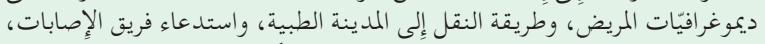

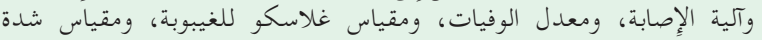

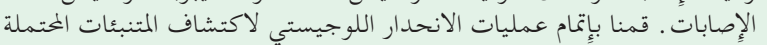

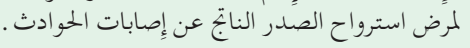

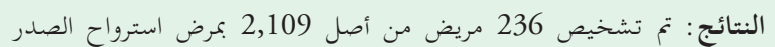

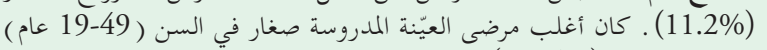

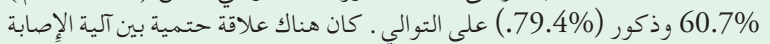

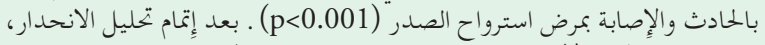

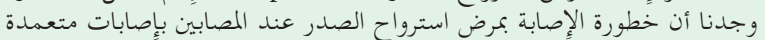

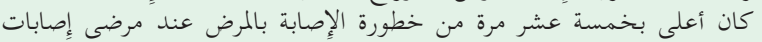

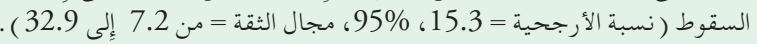

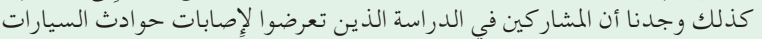

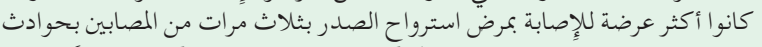

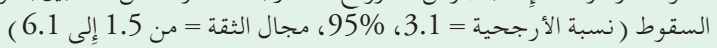

الحلاصة : مرضٍ استرواح الصدر منتشر على مدى كلى كبير ومرتبط مع آلية الإصابة

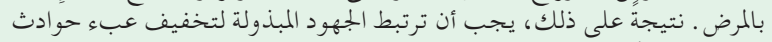

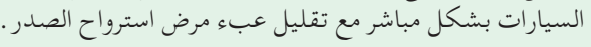

Objectives: To investigate the incidence and predictors of traumatic pneumothorax using data from a Level-I trauma center in Riyadh, Saudi Arabia.

Methods: This is a retrospective chart-review study carried out in King Abdulaziz Medical City, Riyadh, Saudi Arabia. Data were extracted from the hospital's trauma registry (2016-2018). A total of 2,109 trauma patients were included. Inclusion criteria were patients $\geq 16$ years old admitted for a traumatic injury. Variables included patient demographics, transport mode, trauma team activation, mechanism of injury, mortality rate, Glasgow
Coma Scale and Injury Severity Scores. A logistic regression analysis was constructed to evaluate potential predictors of pneumothorax.

Results: Of 2,109 patients included from the trauma registry, 236 (11.2\%) were diagnosed with pneumothorax. The majority of the study population was young (19-49 years) (60.7\%) and male (79.4\%.) Injury mechanism was significantly associated with the presence of pneumothorax $(p<0.001)$. Regression analysis indicated that the odds of having pneumothorax among intentional injury victims was 15 times higher than fall injury victims $(\mathrm{OR}=15.3,95 \% \mathrm{CI}=7.2-32.9)$. Participants who sustained motor vehicle collision injuries had 3 times higher odds of developing pneumothorax than those who suffered fall injuries $(\mathrm{OR}=3.1,95 \% \mathrm{CI}=$ 1.5-6.1).

Conclusion: The incidence of traumatic pneumothorax is sizable and highly associated with the mechanism of injury. Efforts to reduce motor vehicle collision burden should be directly associated with decreasing the burden of traumatic pneumothorax.

Keywords: pneumothorax, chest trauma, prevalence, Saudi Arabia, MVC

Saudi Med J 2020; Vol. 41 (3): 247-252 doi: $10.15537 /$ smj.2020.3.24917

From the Department of Population Health (Alghnam), King Abdullah International Medical Research Center, King Abdulaziz Medical City, National Guard Health Affairs; from the Respiratory Therapy Department (Aldahnim), Specialized Medical Center Hospital; from the College of Medicine (Adebasi), Al Imam Mohammad Ibn Saud Islamic University; from the College of Medicine (Towhari, Alghamdi, Alharbi), King Saud bin Abdulaziz University for Health Sciences; from the Department of Surgery (Almarhabi), King Abdulaziz University; and from the Department of Surgery (Albabtain), King Abdulaziz Medical City, Riyadh, Kingdom of Saudi Arabia.

Received 21st August 2019. Accepted 7th January 2020.

Address correspondence and reprint request to: Dr. Suliman Alghnam, King Abdullah International Medical Research Center, King Abdulaziz Medical City, National Guard Health Affairs, Riyadh, Kingdom of Saudi Arabia.E-mail: Ghnams@ngha.med.sa

ORCID ID: https://orcid.org/0000-0001-5817-0481 
$\mathrm{T}$ raumatic pneumothorax is a potentially lifethreatening condition which occurs when air enters the pleural space as a result of a blunt or penetrating chest injury. ${ }^{1}$ It accounts for one fifth of major trauma victims. ${ }^{2}$ It is a very common cause of chest trauma which can have a good prognosis if recognized and treated early in pre-hospital and emergency department settings. ${ }^{3}$ Vassiliu \& Degiannis ${ }^{4}$ reported that the annual incidence of traumatic pneumothorax is estimated to be 81 per 100,000 people, and the condition accounts for $20.6 \%$ of major trauma patients.

Blunt chest trauma occurs as a result of a blow to the chest or an accident that causes damage to the chest components. It is responsible for approximately $25 \%$ of all traumatic deaths and represents the underlying cause in the vast majority of all traumatic pneumothorax cases. ${ }^{2,5}$ Nevertheless, penetration trauma also has a substantial influence on developing pneumothorax. McPherson et $\mathrm{al}^{6}$ reported that approximately 3 to $4 \%$ of fatally wounded combat casualties died from a tension pneumothorax.

Despite the continuing efforts in advancing trauma management, the overall mortality attributed to chest trauma is as high as $60 \%$ with an incidence accounting for $10 \%$ of trauma admissions. ${ }^{7}$ A study conducted in India ${ }^{8}$ reported that motor vehicle collisions (MVC) accounted for $50 \%$ of chest trauma cases, and $44 \%$ of the thoracic trauma patients were diagnosed with pneumothorax. The predictors of pneumothorax among trauma patients other than MVC include the presence of penetrating chest injuries and rib fractures. It is reported that penetrating injuries have a higher mortality rate compared to blunt trauma. ${ }^{9}$

In Saudi Arabia (SA), trauma continues to be the leading cause of hospitalization. It is estimated that nearly $20 \%$ of The Ministry of Health hospital beds are occupied with MVC trauma patients, ${ }^{10}$ and $80 \%$ of trauma deaths in Saudi hospitals are attributed to MVC cases. ${ }^{11}$

Furthermore, there is a paucity of literature on the incidence of traumatic pneumothorax in SA despite the significance of this condition. A few studies have investigated non-trauma etiologies of pneumothorax in SA. For instance, a study in the Eastern region of the country ${ }^{12}$ found that risk factors for primary spontaneous pneumothorax include being male and a

Disclosure. Authors have no conflict of interests, and the work was not supported or funded by any drug company. smoker; these risk factors are consistent with current literature.

In spite of the extensive international and scattered national reports about traumatic pneumothorax, the incidence and predictors of pneumothorax among trauma patients in SA have not been explored yet. Therefore, we aim to investigate the incidence and determine the predictors of pneumothorax among patients admitted to a major trauma center in Riyadh.

Methods. This is a retrospective chart-review study carried out in King Abdulaziz Medical City (KAMC), Ministry of National Guard Health Affairs, Riyadh, Saudi Arabia. King Abdulaziz Medical City is one of the largest tertiary care centers in SA with a capacity of more than 1500 beds. It is a level-I trauma center which provides complex pre-hospital and trauma emergency services. ${ }^{13}$

This study was reviewed and approved by the Institutional Review Board (IRB) of King Abdullah International Medical Research Center (KAIMRC). Patient confidentiality was ensured throughout the study.

Patients' data were extracted from the trauma registry of KAMC from 2016 to 2018. King Abdulaziz Medical City's trauma registry was established in 2001 by the Department of Surgery to keep track of traumarelated injuries and deaths; it includes i) trauma patients admitted to the hospital through the Emergency Department (ED), ii) trauma patients referred for a particular medical or surgical intervention, iii) trauma patients who were transported to the hospital dead or who died in the hospital. The registry does not include patients who were not admitted to the hospital following ED discharge. Thus, minor trauma cases are not included.

A total of 2,109 trauma patients were included from the registry, 16 years and older, of all nationalities and both genders. Data were validated by trained personnel who checked each patient's electronic medical record.

Study variables included patient's demographics, transport mode (namely via an ambulance or a private car), trauma team activation, mechanism of injury (MVC, motorcycle injury, pedestrian injury, or intentional injury), mortality rate, Glasgow Coma Scale (GCS) and Injury Severity Scores (ISS). Intentional injuries include any violent trauma such as altercation or fights using guns, knives or any sharp objects. Glasgow Coma Scale is a widely and routinely used measure in assessing the level of consciousness of trauma patients; it ranges from 3 (no response at all) to 15 (full response). ${ }^{14}$ Injury Severity Scores is an anatomical scoring system 
for trauma patients used to assess the trauma severity covering 6 body regions; head and neck, face, chest, abdomen, extremities and external. ${ }^{15}$ Pneumothorax diagnosis was verified by a respiratory therapist by reviewing all medical records of included patients.

Statistical analysis. The data were analyzed using STATA version 14, College Station, TX: StataCorp LP. The analysis included descriptive and analytic statistics. Data were expressed as mean and standard deviation (SD) for continuous variables (age, weight, ISS, and GCS). Percentages were used to describe categorical variables (gender, death rates, injury mechanism, and transportation mode). A Chi-square test was performed to compare categorical variables and a t-test for continuous variables. A $p \leq 0.05$ was considered statistically significant. A univariate regression analysis was conducted for the pneumothorax mechanism of injury in the first model. This analysis was followed by multivariable logistic regression analysis in the second model. Possible confounders that were adjusted for were age (0-18 years as a reference), gender (female as a reference), mechanism of injury (falls as a reference), transport mode (ambulance as a reference) and ISS (scores of 0-14 as a reference). Selection of these variables was based on previous literature and their association with trauma outcomes. Odds ratio was reported with an associated $95 \%$ confidence interval. Model discrimination was evaluated by the area under the curve and goodness of fit by the Hosmer-Lemeshow. The variance inflation factor was used to evaluate multicollinearity among variables.

Results. Table 1 summarizes the descriptive characteristics and demographics of the study population. Of 2,109 trauma patients, 236 (11.2\%) were diagnosed with pneumothorax. The majority of them were young (19-49 years) $60.7 \%$ and male $79.4 \%$. Approximately half of the study population (52.6\%) was transported via an ambulance. The mechanism of injury was significantly associated with the presence of pneumothorax $(p<0.001)$. In the pneumothorax group, the most frequent mechanism of injury leading to pneumothorax was MVC (69.1\%), followed by intentional injuries (16.5\%) and pedestrian accidents $(6.8 \%)$. Patients with pneumothorax sustained more severe injuries based on the ISS (median: 18 versus 9, $p<0.001$ ). About a third of the subjects had upper limb

Table 1 - Descriptive characteristics and demographics of patients with and without pneumothorax following injury in Riyadh (2016-2018).

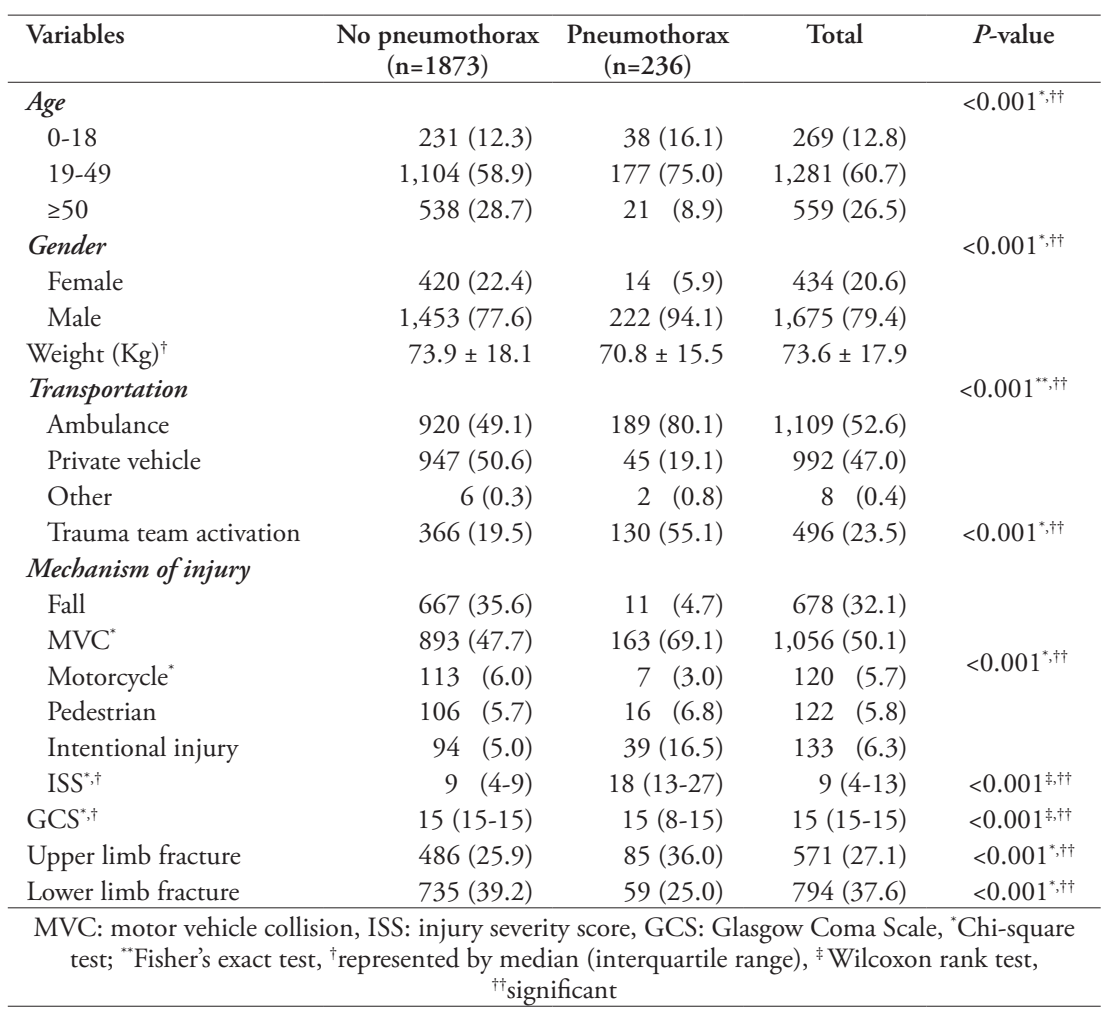


fractures alongside pneumothorax (36\%, $p<0.001)$. Approximately tenth (10.6\%) of the pneumothorax group died.

A logistic regression model of the association between pneumothorax and other variables is shown in Table 2. The multivariable logistic regression analysis indicates that the odds of having pneumothorax among older adults ( $\geq 50$ years) was lower than the pediatric age group $(\mathrm{OR}=0.5,95 \% \mathrm{CI}=0.3-1.0)$. Males had twice the odds of developing pneumothorax than females adjusting for the covariates $(\mathrm{OR}=1.9 .95 \% \mathrm{CI}=1.0-3.4)$. There is a significant association between the mechanism of injury and pneumothorax. Intentional injury victims, those who suffered penetrating injuries, had 15 times higher odds of developing pneumothorax than those who suffered fall injuries $(\mathrm{OR}=15.3,95 \% \mathrm{CI}=7.2-32.9)$. Participants who sustained MVC-related injuries had 3 times higher odds of developing pneumothorax than the reference group $(\mathrm{OR}=3.1,95 \% \mathrm{CI}=1.5-6.1)$. This analysis also found that patients, who sustained severe injuries, had 7 times higher odds of developing pneumothorax than those who did not suffer severe injuries $(\mathrm{OR}=7.2,95 \% \mathrm{CI}=4.9-10.4)$.

Discussion. This study investigated the incidence and predictors of traumatic pneumothorax among patients admitted to a level-I trauma center in Riyadh. The study found that one in 10 trauma patients developed pneumothorax. This finding highlights a sizable incidence of pneumothorax among trauma patients, especially those who suffered multiple injuries. Furthermore, the finding emphasizes the burden of traumatic pneumothorax adds to the healthcare system and necessities a major need to invest in populationbased primary prevention to improve population health and reduce injuries.

Due to the paucity of literature on the incidence of traumatic pneumothorax in SA, it is difficult to compare study findings with other local studies. However, the incidence found in several previous international reports is higher than the current finding. Di Bartolomeo et $\mathrm{al}^{2}$ reported that the incidence of traumatic pneumothorax in Italy is $20 \%$, and Oguns ${ }^{16}$ found an incidence of 28\% in Queensland, Australia. In contrast, Veysi et $\mathrm{al}^{7}$ reported that the incidence of traumatic pneumothorax in the United Kingdom is $4.5 \%$. This study should accordingly estimate the incidence of traumatic pneumothorax in SA and provide the basis of comparison for future research in this area.

The present study also shows that MVC accounted for more than two-thirds of the traumatic pneumothorax cases, followed by intentional injuries which were responsible for $16.5 \%$ of the cases. Several studies ${ }^{2,17}$ support this finding that the leading cause of traumatic pneumothorax is MVC. The most common mechanism of injury was a vehicle rolling over and causing a blunt

Table 2 - A logistic regression model of the association between pneumothorax and other variables of interest.

\begin{tabular}{|c|c|c|c|c|}
\hline \multirow[t]{2}{*}{ Variables } & \multicolumn{2}{|c|}{ Unadjusted model } & \multicolumn{2}{|c|}{ Adjusted model ${ }^{*}$} \\
\hline & $\mathrm{OR}^{*}$ & $95 \% \mathrm{CI}$ & OR & $95 \% \mathrm{CI}$ \\
\hline \multicolumn{5}{|l|}{ Age } \\
\hline $0-18$ & Reference & & Reference & \\
\hline $19-49$ & 1.0 & $0.6-1.4$ & 0.8 & $0.5-1.3$ \\
\hline$\geq 50$ & 0.3 & $0.2-0.5$ & 0.5 & $0.3-1.0$ \\
\hline \multicolumn{5}{|l|}{ Gender } \\
\hline Male & 4.6 & $2.6-7.9$ & 1.9 & $1.0-3.4$ \\
\hline \multicolumn{5}{|l|}{ Mechanism of injury } \\
\hline Falls & Reference & & Reference & \\
\hline MVC & 11.1 & $5.9-20.5$ & 3.1 & $1.5-6.1$ \\
\hline Motorcycle & 3.7 & $1.4-9.9$ & 1.4 & $0.5-4.1$ \\
\hline Pedestrian & 9.1 & $4.1-20.2$ & 1.9 & $0.8-4.6$ \\
\hline Intentional injury & 25.1 & $12.4-50.8$ & 15.3 & $7.2-32.9$ \\
\hline \multicolumn{5}{|l|}{ Transportation } \\
\hline Ambulance & Reference & & Reference & \\
\hline Private vehicle & 0.2 & $0.2-0.3$ & 0.8 & $0.5-1.2$ \\
\hline Other & 1.7 & $0.2-8.6$ & 1.6 & $0.3-9.4$ \\
\hline \multicolumn{5}{|l|}{ ISS } \\
\hline $0-14$ & Reference & & Reference & \\
\hline $15-75$ & 8.4 & $6.3-11.2$ & 7.2 & $4.9-10.4$ \\
\hline
\end{tabular}


or penetrating chest injury. Penetrating injuries and rib fractures were consistent with an ejection of the victim from the vehicle. This implies, not surprisingly, a low compliance of seatbelt use by drivers in our region. We were unable to assess that in our study because seatbelt use was documented in less than $5 \%$ of the patients in the registry. Alghnam et $\mathrm{al}^{18}$ reported in his observational study that only a third of the drivers in Riyadh, SA wear seatbelts. Hence, these findings underline the burden that MVC pose on patients' outcomes and healthcare facilities and call for an intervention to improve traffic safety in SA.

Based on the ISS, patients with pneumothorax suffered more severe injuries than patients without pneumothorax, and approximately $10 \%$ of the patients in the traumatic pneumothorax group died. This highlights the significance of directing resources toward early recognition and intervention of traumatic pneumothorax in order to prevent associated deaths. Hence, this study should contribute highly to the success of Saudi Vision 2030. This initiative is a national blueprint founded to achieve 96 strategic objectives in order to develop public service sectors including healthcare. One of the goals of Vision 2030 is to enhance the quality of preventive and therapeutic healthcare services by providing physicians with enhanced training to advance the management and treatment of threatening conditions like traumatic pneumothorax. By doing so, they can help reduce the high mortality rate associated with pneumothorax. ${ }^{19}$

Another notable finding in this study is that males had higher odds of developing pneumothorax, explained by the fact that $94.1 \%$ of the injured population was male. This is mainly because females were not allowed to drive in SA during the study period. Since females are currently allowed to drive in SA, further research should be carried out with the aim of finding out if the incidence of traumatic pneumothorax will be different due to the new driving laws in SA. Moreover, approximately three-quarters of the population was between 19 and 49 years old. This is explained by the fact that the majority of the population in SA is young with a median age of 30 years. This finding should serve as a guide when choosing the target population for preventive programs and awareness campaigns.

The current study found that in approximately $80 \%$ of the patients who developed pneumothorax, the cause was blunt chest injury due to MVC as the primary underlying cause. These findings are supported in literature ${ }^{10,20}$ where MVC is the underlying cause in the majority of the blunt chest injuries. However, victims of intentional injuries, who sustained penetrating chest wounds, had 15 times higher odds of developing pneumothorax than non-intentional injury victims with blunt chest injury. Penetrating injuries result in a direct damage to the lung parenchyma and pleura causing the pleural space to be open to the atmosphere, and resulting in a partially or completely collapsed lung. The damage to the chest tissue and organs caused by the penetrating chest injuries explains why this type of trauma accounts for higher morbidity and mortality rates than blunt chest injuries. ${ }^{9}$ Further research is needed to explore the causes and predictors of pneumothorax due to penetrating chest injuries in SA.

The current findings should serve as the basis for future research about the incidence, predictors, management and prevention of traumatic pneumothorax in SA and other developing countries where research about traumatic pneumothorax is scarce. Another addition of this study to literature is that it provides an estimation of the incidence of traumatic pneumothorax in the community, raising awareness on the seriousness of this condition and helping find preventive strategies to reduce its occurrence. Finally, the data of this study were validated through checking each patient's electronic medical record by a respiratory therapist to insure the accuracy of the data obtained.

Study limitations. There are a number of limitations that must be acknowledged in this study. The current findings are based on data from a single hospital and cannot be generalized to the whole population of Riyadh. Another limitation is that the diagnosis of pneumothorax was made using plain radiology. This may be important as some of the patients with chest injuries may develop occult pneumothorax that can only be detected through computed tomography (CT). Occult pneumothorax accounts for $2-15 \%$ of polytrauma patients. ${ }^{21}$ Thus, the actual incidence of pneumothorax may have been underestimated.

In conclusion, the findings of our study are consistent with current international literature about the incidence and predictors of traumatic pneumothorax. These findings should add substantially to literature as they will provide the basis for research on the incidence, predictors, management and prevention of traumatic pneumothorax in SA and other developing countries. Preventive measures and heightened awareness should be considered as they may reduce the burden of traumatic pneumothorax in SA.

Acknowledgment. The authors would like to thank www.escienta.com for the English language editing. 


\section{References}

1. Bintcliffe O, Maskell N. Spontaneous pneumothorax. BMJ 2014; 348: g2928.

2. Di Bartolomeo S, Sanson G, Nardi G, Scian F, Michelutto V, Lattuada L. A population-based study on pneumothorax in severely traumatized patients. J Trauma 2001; 51: 677-682.

3. Mennicke M, Gulati K, Oliva I, Goldflam K, Skali H, Ledbetter $\mathrm{S}$, et al. Anatomical distribution of traumatic pneumothoraces on chest computed tomography: implications for ultrasound screening in the ED. The American Journal of Emergency Medicine 2012; 30: 1026.

4. Vassiliu P., Degiannis E. Hemothorax, Pneumothorax, and Chest Empyema. In: Moore L, Todd S, editors. Common problems in acute care surgery. 2nd ed. Cham (SW): Springer International Publishing; 2017.

5. El-Menyar A, Abdelrahman $\mathrm{H}$, Al-Hassani A, Ellabib M, Asim M, Zarour A, et al. Clinical presentation and time-based mortality in patients with chest injuries associated with road traffic accidents. Arch Trauma Res 2016; 5: 1.

6. McPherson JJ, Feigin DS, Bellamy RF. Incidence of Tension Pneumothorax in Fatally Wounded Combat Casualties. J Trauma Inj Infect Crit Care 2006; 60: 573-578.

7. Veysi VT, Nikolaou VS, Paliobeis C, Efstathopoulos N, Giannoudis P V. Incidence of chest trauma, associated injuries and mortality: a level I trauma centre experience. Int Orthop 2009; 33: 1425-1433.

8. Kumar D, Chakravarthy D, Bharath D. Blunt Trauma Chest: A Study on Clinical Pattern. IOSR Journal of Dental and Medical Sciences 2017; 16: 2-4.

9. Edgecombe L, Angus L. Thoracic Trauma. Treasure Island (FL): StatPearls Publishing; 2019.

10. Hokkam E, Gonna A, Zakaria O, El-shemally A. Trauma patterns in patients attending the Emergency Department of Jazan General Hospital, Saudi Arabia. World J Emerg Med 2015; 6: 49-50.
11. Alghnam S, Alkelya M, Al-Bedah K, Al-Enazi S. Burden of traumatic injuries in Saudi Arabia: lessons from a major trauma registry in Riyadh, Saudi Arabia. Ann Saudi Med 2014; 34: 293.

12. Aljehani Y, Almajid F, Niaz R, Elghoneimy Y. Management of primary spontaneous pneumothorax: A single-center experience. Saudi J Med Med Sci 2018; 6: 102-103.

13. Ministry of National Guard - Health Affairs. King Abdulaziz Medical City in Riyadh. [Updated 2016 Aug 24; Accessed 2020 January 1]. Available from: https://ngha.med.sa/English/ MedicalCities/AlRiyadh/Pages/default.aspx

14. BrainLine. What is the glasgow coma scale? [Update 2018 July 25, Access 2020 January 1]. Available from: https://www. brainline.org/article/what-glasgow-coma-scale

15. Agency for Clinical Innovation. Injury severity score. [Update 2008; Accessed 2020 January 1]. Available from: https://www. aci.health.nsw.gov.au/get-involved/institute-of-trauma-andinjury-management/Data/injury-scoring/injury_severity_score

16. Oguns OJ. Predictors of pneumothorax in motor vehicle accident (MVA) survivors who sustain chest trauma. [Update 2014 February; cited 2018 August 9]; Available from: http:// scholar.sun.ac.za/handle/10019.1/99740

17. Zhang S, Tang M, Ma J, Yang J, Qin X, Jin W et al. Thoracic trauma: a descriptive review of 4168 consecutive cases in East China. Medicine 2019; 98: 2-4.

18. Alghnam S, Alrowaily M, Alkelya M, Alsaif A, Almoaiqel F, Aldegheishem A. The incidence of seatbelt and mobile phone use among drivers in Riyadh, Saudi Arabia: An observational study. J Safety Res 2018; 66: 33-37.

19. Saudi Vision 2030. National Transformation Program. [Updated 2019; cited 2019 July 9]. Available from: https://vision2030. gov.sa/en

20. Wang X, Karki A, Du D, Zhao X, Xiang X, Lu Z. Plasma levels of high mobility group box 1 increase in patients with posttraumatic stress disorder after severe blunt chest trauma: a prospective cohort study. Surg Res 2015; 193: 309.

21. Llaquet Bayo H, Montmany Vioque S, Rebasa P, Navarro Soto S. Results of conservative treatment in patients with occult pneumothorax. Cirugía Española 2016; 94: 233. Spanish 